\title{
Treatment Delay in Melanoma: A Risk Factor Analysis of an Impending Crisis
}

\author{
Marcus L. Elias, MD; Ann M. John, MD; Spandana Maddukuri, BS; Robert A. Schwartz, MD, MPH; \\ W. Clark Lambert, MD, PhD
}

\section{PRACTICE POINTS}

- Melanoma treatment delays (MTDs) have been linked to poor outcomes.

- Based on the National Cancer Database, the mean MTD has increased significantly from 2004 to 2015 $(P<.001)$.

- More delays are seen in patients who are older than 50 years, female, nonwhite, not privately insured, and treated at an academic facility and who have more advanced tumor stage and head/neck primaries.

Risk factors associated with melanoma treatment delay (MTD) have been inadequately studied. To elucidate MTD associations based on patient and tumor characteristics, a retrospective cohort study was performed for cutaneous melanoma cases reported to the National Cancer Database (NCDB) between 2004 and 2015. We evaluated the number of days from diagnosis to treatment initiation, analyzing postponements more than 45 days as moderate MTD (mMTD) and postponements more than 90 days as severe MTD (sMTD). Greater MTD rates were independently associated with patients who are older than 50 years, female, nonwhite, not privately insured, and treated at an academic facility and who have more advanced tumor stage and head/neck primaries.

Cutis. 2021;107:E19-E26.

M elanoma is the most lethal skin cancer and is the second most common cancer in adolescents and young adults. ${ }^{1}$ It is the fifth most common cancer in the United States based on incidence, which has steadily risen for the last 2 decades. ${ }^{2,3}$ For melanoma management, delayed initial diagnosis has been associated with more advanced lesions at presentation and poorer outcomes. ${ }^{4}$ However, the prognostic implications of delaying melanoma management after diagnosis merits further scrutiny.

This study investigates the associations between melanoma treatment delay (MTD) and patient and tumor characteristics. Although most cases undergo surgical treatment first, more advanced stages may require initiating chemotherapy, radiation therapy, or immunotherapy. In addition, patients who are poor surgical candidates may opt for topical field therapy, such as imiquimod for superficial lesions, prior to more definitive treatment. ${ }^{5}$ In the Medicaid population, patients who are older than 85 years, married, and previously diagnosed with another melanoma and who also have an increased comorbidity burden have a higher likelihood of MTD. ${ }^{6}$ For nonmelanoma skin cancers, patient denial is the most common patient-specific factor accounting for treatment delay. ${ }^{7}$ For this study, our aim was to further evaluate the independent risk factors associated with MTD.

\section{Methods}

Case Selection-The National Cancer Database (NCDB) was queried for all cutaneous melanoma cases from 2004 to $2015(\mathrm{~N}=525,271)$. The NCDB is an oncology database sourced from more than 1500 accredited cancer facilities in the United States and Puerto Rico. It receives cases from academic hospitals, Veterans Health Administration hospitals, and community centers. ${ }^{8}$ Annually, the database collects approximately $70 \%$ of cancer diagnoses and $48 \%$ of melanoma diagnoses in the United States. ${ }^{9,10}$ Per institutional guidelines, this analysis was determined to be exempt from institutional

Drs. Elias, Schwartz, and Lambert are from the Division of Dermatology, Rutgers New Jersey Medical School, Newark. Dr. Lambert also is from the Department of Pathology, Immunology, and Laboratory Medicine. Dr. John is from the Department of Dermatology, Rutgers Robert Wood Johnson Medical School, Piscataway, New Jersey. 
review board approval due to the deidentified nature of the dataset.

The selection scheme is illustrated in Table 1. International Statistical Classification of Diseases and Related Health Problems histology codes 8720/3 through 8780/3 combined with the site and morphology primary codes C44.0 through C44.9 identified all patients with a diagnosis of cutaneous melanoma. Primary site was established with the histology codes in the following manner: C44.0 through C44.4 for head/neck primary, C44.5 for trunk primary, C44.6 through C44.7 for extremity primary, and C44.8 through C44.9 for not otherwise specified. Because the NCDB does not specify cause of death, any cases in which the melanoma diagnosis was not the patient's primary (or first) cancer diagnosis were excluded because of potential ambiguity. Cases lacking histologic confirmation of the diagnosis after primary site biopsy or cases diagnosed from autopsy reports also were excluded. Reports missing staging data or undergoing palliative management were removed. In total, 104,118 cases met the inclusion criteria.

Variables of Interest-The NCDB database codes for a variable "Treatment Started, Days from Dx" are defined as the number of days between the date of diagnosis and the date on which treatment-surgery, radiation, systemic, or other therapy - of the patient began at any facility. ${ }^{11}$ Treatment delays were classified as more than 45 days or more than 90 days. These thresholds were chosen based on previous studies citing a 45-day recommendation as the timeframe in which primary site excision of melanoma should occur for improved outcomes. . $^{1,6,12}$ Additionally, the postponement cutoffs were aligned with prior studies on surgical delay in melanoma for the Medicaid population. ${ }^{6}$ Delays of 45 days were labeled as moderate MTD (mMTD), whereas postponements more than 90 days were designated as severe MTD (sMTD).

\section{TABLE 1. Inclusion Criteria}

\begin{tabular}{lll}
\hline Exclusion & No. excluded & No. remaining \\
\hline $\begin{array}{l}2004-2015 \\
\text { cutaneous } \\
\text { melanoma cases }\end{array}$ & 525,271 \\
\hline $\begin{array}{l}\text { Nonprimary } \\
\text { malignancies }\end{array}$ & 147,024 & 378,247 \\
\hline $\begin{array}{l}\text { Dead at O months } \\
\text { (diagnosis at }\end{array}$ & 661 & 377,586 \\
\begin{tabular}{l} 
autopsy) \\
\hline $\begin{array}{l}\text { Missing } \\
\text { staging data }\end{array}$
\end{tabular} & 327,639 \\
\hline $\begin{array}{l}\text { No biopsy of } \\
\text { the primary site/ } \\
\text { missing histologic } \\
\text { confirmation }\end{array}$ & 222,952 & 104,687 \\
\hline Palliative care & 569 & \\
\hline \begin{tabular}{l} 
Study population \\
\hline
\end{tabular} & & 104,118 \\
\hline
\end{tabular}

Patient and tumor characteristics were analyzed for associations with MTD (Table 2). Covariates included age, sex, race (white vs nonwhite), Hispanic ethnicity, insurance status (private; Medicare, Medicaid or other government insurance; and no insurance), median annual income of the patient's residential zip code (based on 2008-2012 census data), percentage of the population of the patient's residential zip code without a high school degree (based on 2008-2012 census data), Charlson-Deyo (CD) comorbidity score (a weighted score derived from the sum scores for comorbid conditions), geographic location (rural, urban, and metropolitan), and treatment facility (academic vs nonacademic). Tumor characteristics included primary site (head/neck, trunk, and extremities), stage, and Breslow depth of invasion. Tumor stage was determined using the American Joint Committee on Cancer 6th and 7th editions, depending on the patient's year of diagnosis.

Statistical Methods $-\chi^{2}$ and Fisher exact tests were used to analyze categorical variables involving patient demographics and tumor characteristics by bivariate analysis (Tables 3 and 4). Multivariate analysis determined the relative impact on MTD by including variables that significantly differed on bivariate $\chi^{2}$ analysis (Table 2). Multivariate modeling determined odds ratio (OR) and corresponding 95\% CI for the risk-adjusted associations of the variables with MTD. All statistical analyses were performed using SPSS Statistics version 23 (IBM). $P<.05$ was considered statistically significant, and all statistical tests were 2-tailed. Line graph figures by year of diagnosis were modeled by SPSS using the mean days of delay per year. Independent sample $t$ tests assessed for differences in mean values.

\section{Results}

The final study population included 104,118 patients, most of whom were male $(56.4 \%)$, white $(96.6 \%)$, and aged 50 to 74 years (54.4\%). Most patients were privately insured $(52.6 \%)$, had no CD comorbidities $(87.5 \%)$, and lived in metropolitan cities (80.4\%)(Table 3). A large majority $(95,473$ [91.7\%]) of patients received surgery as the first means of treatment, with a smaller portion (863 [0.8\%]) having unspecified systemic therapy first. The remaining cases were first treated with chemotherapy (1738 [1.7\%]), immunotherapy (382 [0.4\%]), or radiation $(490[0.5 \%])$, and the rest did not specify treatment sequence. The tumors were most commonly located on the extremities $(40.7 \%)$, were stage I $(41.2 \%)$, and had a Breslow depth of less than $1 \mathrm{~mm}(41.6 \%)$.

Treatment delay averaged 31.55 days, with a median of 27 days. Overall mean MTD increased significantly from 29.74 days in 2004 to 32.55 days in 2015 (2-tailed $t$ test; $P<.001$ )(Figure). A total of 78,957 cases $(75.8 \%)$ received treatment within 45 days, whereas 2467 cases $(2.5 \%)$ were postponed past 90 days. On bivariate analysis, age, sex, race, insurance status, Hispanic 
TABLE 2. Multivariate Analysis of Patient and Tumor-Related Associations With Melanoma Treatment Delay ${ }^{a}$

\begin{tabular}{|c|c|c|c|c|c|c|}
\hline \multirow[b]{2}{*}{ Patient characteristic } & \multicolumn{3}{|c|}{$>45$ days (moderate delay) } & \multicolumn{3}{|c|}{$>90$ days (severe delay) } \\
\hline & OR & $95 \% \mathrm{Cl}$ & $P$ value & OR & $95 \% \mathrm{Cl}$ & $P$ value \\
\hline \multicolumn{7}{|l|}{ Age,y } \\
\hline \multicolumn{7}{|l|}{$<50^{\mathrm{b}}$} \\
\hline $50-74$ & 1.072 & $1.007-1.141$ & .029 & 1.147 & $0.975-1.349$ & .098 \\
\hline$\geq 75$ & 1.278 & $1.184-1.380$ & $<.001$ & 1.590 & $1.313-1.925$ & $<.001$ \\
\hline \multicolumn{7}{|l|}{ Sex } \\
\hline \multicolumn{7}{|l|}{ Male $^{b}$} \\
\hline Female & 1.052 & $1.011-1.095$ & .013 & .965 & $0.874-1.066$ & .483 \\
\hline \multicolumn{7}{|l|}{ Race } \\
\hline \multicolumn{7}{|l|}{ White ${ }^{b}$} \\
\hline Nonwhite & 1.405 & $1.245-1.585$ & $<.001$ & 1.674 & $1.315-2.131$ & $<.001$ \\
\hline \multicolumn{7}{|l|}{ Non-Hispanic ${ }^{b}$} \\
\hline Hispanic & 1.809 & $1.587-2.063$ & $<.001$ & 2.749 & $2.198-3.439$ & $<.001$ \\
\hline \multicolumn{7}{|l|}{ Insurance status } \\
\hline \multicolumn{7}{|l|}{ Private $^{b}$} \\
\hline Medicare & 1.054 & $1.001-1.111$ & .046 & 1.065 & $0.946-1.199$ & .300 \\
\hline Medicaid/other government insurance & 1.668 & $1.509-1.843$ & $<.001$ & 2.336 & $1.919-2.845$ & $<.001$ \\
\hline No insurance & 1.642 & $1.457-1$ & $<.001$ & 2.582 & $2.061-3.234$ & $<.001$ \\
\hline \multicolumn{7}{|l|}{ Median annual income, \$ } \\
\hline \multicolumn{7}{|l|}{$<38,000^{b}$} \\
\hline $38,000-47,999$ & 0.951 & $0.875-1.033$ & .232 & 0.844 & $0.712-1.002$ & .052 \\
\hline $48,000-62,999$ & 0.934 & $0.861-1.013$ & .097 & 0.829 & $0.694-0.989$ & .038 \\
\hline$\geq 63,000$ & 048 & $0.961-1.144$ & .290 & 1.068 & $0.884-1.290$ & .496 \\
\hline \multicolumn{7}{|l|}{$\begin{array}{l}\text { Percentage of population of } \\
\text { patient's residential zip code } \\
\text { without high school degree } \\
\quad<7^{\text {b }}\end{array}$} \\
\hline $7-12.9$ & 1.285 & $1.183-1.396$ & $<.001$ & 1.383 & $1.130-1.693$ & .002 \\
\hline $13-20.9$ & 1.238 & $1.167-1.314$ & $<.001$ & 1.312 & $1.117-1.541$ & .001 \\
\hline$\geq 21$ & 1.107 & $1.052-1.165$ & $<.001$ & 1.219 & $1.072-1.385$ & .002 \\
\hline \multicolumn{7}{|l|}{$\begin{array}{l}\text { Charlson-Deyo } \\
\text { comorbidity score } \\
\mathrm{O}^{\mathrm{b}}\end{array}$} \\
\hline 1 & 1.080 & $1.018-1.147$ & .011 & 1.073 & $0.930-1.237$ & .333 \\
\hline$\geq 2$ & 1.364 & $1.217-1.529$ & $<.001$ & 1.877 & $1.499-2.351$ & $<.001$ \\
\hline \multicolumn{7}{|l|}{ Treatment facility } \\
\hline \multicolumn{7}{|l|}{ Nonacademic ${ }^{b}$} \\
\hline Academic & 1.578 & $1.518-1.640$ & $<.001$ & 1.366 & $1.242-1.502$ & $<.001$ \\
\hline \multicolumn{7}{|l|}{ Primary site } \\
\hline \multicolumn{7}{|l|}{ Head/neck ${ }^{b}$} \\
\hline Trunk & 0.620 & $0.589-0.654$ & $<.001$ & 0.540 & $0.472-0.618$ & $<.001$ \\
\hline Extremities & 0.641 & $0.613-0.672$ & $<.001$ & 0.632 & $0.565-0.706$ & $<.001$ \\
\hline
\end{tabular}


TABLE 2. (continued)

\begin{tabular}{|c|c|c|c|c|c|c|}
\hline \multirow[b]{2}{*}{ Patient characteristic } & \multicolumn{3}{|c|}{$>45$ days (moderate delay) } & \multicolumn{3}{|c|}{$>90$ days (severe delay) } \\
\hline & OR & $95 \% \mathrm{Cl}$ & $P$ value & OR & $95 \% \mathrm{Cl}$ & $P$ value \\
\hline \multicolumn{7}{|l|}{$\overline{\text { Stage }}$} \\
\hline \multicolumn{7}{|l|}{ In situ } \\
\hline I & 0.902 & $0.861-0.945$ & $<.001$ & 0.690 & $0.613-0.778$ & $<.001$ \\
\hline$\|$ & 1.130 & $1.065-1.199$ & $<.001$ & 1.085 & $0.946-1.244$ & .244 \\
\hline III & 1.196 & $1.116-1.282$ & $<.001$ & 1.204 & $1.026-1.412$ & .023 \\
\hline IV & 1.690 & $1.477-1.934$ & $<.001$ & 2.240 & $1.779-2.820$ & $<.001$ \\
\hline
\end{tabular}

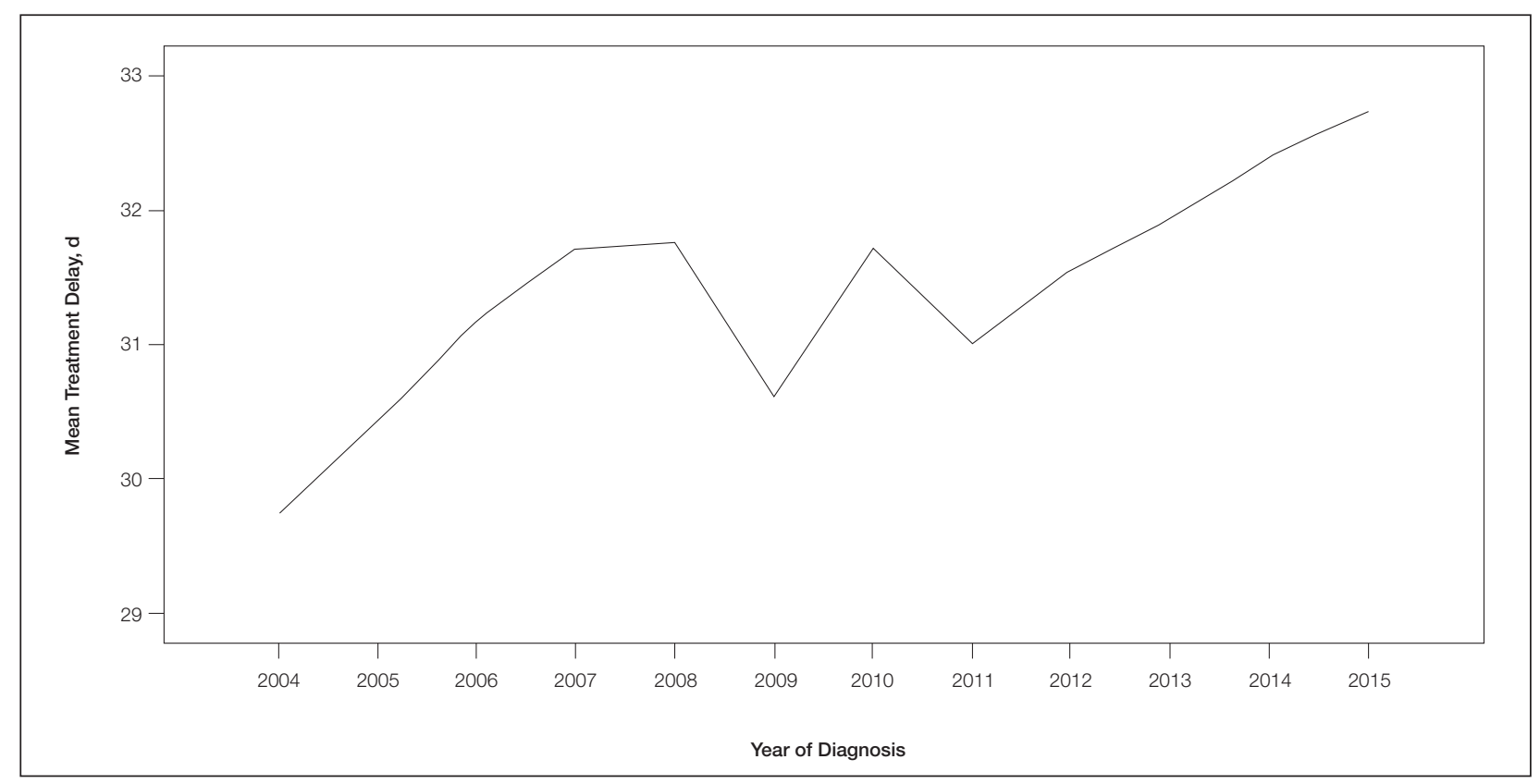

Overall trend of melanoma treatment delay (2004-2015).

ethnicity, median annual income of residential zip code, percentage of the population of the patient's residential zip code with high school degrees, CD score, and academic treatment facility held significant associations with mMTD and sMTD $(P<.05)$ (Table 3$)$. Analyzing bivariate associations with pertinent tumor characteristicsprimary site, stage, and Breslow depth-also held significant associations with MMTD and sMTD $(P<.001)$ (Table 4).

On multivariate analysis, controlling for the variables significant on bivariate analysis, multiple factors showed independent associations with MTD (Table 2). Patients aged 50 to 74 years were more likely to have mMTD (reference: $<50$ years; $P=.029 ; \mathrm{OR}=1.072$ ). Patients 75 years and older showed greater rates of mMTD (reference: $<50$ years; $P<.001 ; \mathrm{OR}=1.278$ ) and $\mathrm{sMTD}$ $(P<.001 ; \mathrm{OR}=1.590)$. Women had more mMTD $(P=.013$;
$\mathrm{OR}=1.052$ ). Nonwhite patients had greater rates of both mMTD (reference: white; $P<.001 ; O R=1.405$ ) and sMTD $(P<.001 ; \mathrm{OR}=1.674)$. Hispanic patients also had greater mMTD (reference: non-Hispanic: $P<.001 ; \mathrm{OR}=1.809$ ) and $\operatorname{sMTD}(P<.001 ; \mathrm{OR}=2.749)$. Compared to patients with private insurance, those with Medicare were more likely to have mMTD $(P=.046 ; \mathrm{OR}=1.054)$. Patients with no insurance or Medicaid/other government insurance showed more mMTD (no insurance: $P<.001$, $\mathrm{OR}=1.642$; Medicaid/other: $P<.001, \mathrm{OR}=1.668$ ) and sMTD (no insurance: $P<.001, \mathrm{OR}=2.582$; Medicaid/other: $P<.001$, $\mathrm{OR}=2.336$ ) .

With respect to the median annual income of the patient's residential zip code, patients residing in areas with a median income of $\$ 48,000$ to $\$ 62,999$ were less likely to have an sMTD (reference: $<\$ 38,000 ; P=.038$; 
TABLE 3. Univariate $\chi^{2}$ Analysis of Patient Characteristics and Melanoma Treatment Delay

\begin{tabular}{|c|c|c|c|c|c|c|}
\hline \multirow[b]{2}{*}{ Patient characteristic } & \multicolumn{6}{|c|}{ Frequency, n (\%) } \\
\hline & $\begin{array}{l}\text { All cases } \\
(\mathrm{N}=104,118)\end{array}$ & $\begin{array}{l}\text { Delay of } \\
\text { treatment, } \leq 45 \mathrm{~d} \\
(\mathrm{n}=78,957)\end{array}$ & $\begin{array}{l}\text { Delay of } \\
\text { treatment, }>45 \\
d(n=17,681)\end{array}$ & $P$ value & $\begin{array}{l}\text { Delay of } \\
\text { treatment, }>90 \\
d(n=2467)\end{array}$ & $P$ value \\
\hline Age, y & & & & $<.001$ & & $<.001$ \\
\hline$<50$ & 23,737 (22.8) & $18,730(84.1)$ & 3548 (15.9) & & $466(2.1)$ & \\
\hline $50-74$ & $56,640(54.4)$ & $43,311(82.1)$ & $9427(17.9)$ & & $1245(2.3)$ & \\
\hline$\geq 75$ & $23,741(22.8)$ & $16,916(78.2)$ & $4706(21.8)$ & & $756(3.5)$ & \\
\hline Sex & & & & $<.001$ & & .005 \\
\hline Male & $58,723(56.4)$ & $44,170(81.2)$ & 10,209 (18.8) & & $1458(2.7)$ & \\
\hline Female & 45,395 (43.6) & 34,787 (82.3) & $7472(17.7)$ & & $1009(2.4)$ & \\
\hline Race & & & & $<.001$ & & $<.001$ \\
\hline White & $100,578(96.6)$ & 76,461 (81.9) & $16,947(18.1)$ & & $2328(2.5)$ & \\
\hline Nonwhite & $3540(3.4)$ & 2496 (77.3) & $734(22.7)$ & & $139(4.3)$ & \\
\hline Insurance status ${ }^{a}$ & & & & & & $<.001$ \\
\hline Private & $55,074(52.6)$ & $43,029(83.8)$ & $8304(16.2)$ & & $992(1.9)$ & \\
\hline Medicare & $41,265(40.2)$ & $30,228(79.9)$ & $7609(20.1)$ & 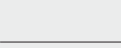 & $1103(2.9)$ & \\
\hline $\begin{array}{l}\text { Medicaid/other } \\
\text { government insurance }\end{array}$ & 3721 (3.6) & $2445(72.7)$ & $917(27.3)$ & & $192(5.6)$ & \\
\hline No insurance & $2644(2.5)$ & 1760 (75.2) & $580(24.8)$ & & $134(5.6)$ & \\
\hline Hispanic ethnicity & & $1078(68.7)$ & 491 (31.3) & $<.001$ & $139(8.8)$ & $<.001$ \\
\hline Median annual income, $\$$ & & & & $<.001$ & & $<.001$ \\
\hline$<38,000$ & $11,298(10.9)$ & $8331(80.1)$ & 2074 (19.9) & & $349(3.3)$ & \\
\hline $38,000-47,999$ & $21,426(20.6)$ & $16,214(81.3)$ & $3723(18.7)$ & & $512(2.6)$ & \\
\hline 48,000-62,999 & $28,593(27.5)$ & $21,882(82.3)$ & $4693(17.7)$ & & $614(2.3)$ & \\
\hline$\geq 63,000$ & $42,495(40.8)$ & $31,903(81.9)$ & $7031(18.1)$ & & $967(2.5)$ & \\
\hline $\begin{array}{l}\text { Percentage of population of } \\
\text { patient's residential zip code } \\
\text { without high school degree }\end{array}$ & & & & $<.001$ & & $<.001$ \\
\hline$<7$ & 34,149 (32.8) & 26,502 (83.6) & $5184(16.4)$ & & $687(2.2)$ & \\
\hline $7-12.9$ & $36,506(35.1)$ & $27,581(81.5)$ & 6255 (18.5) & & $836(2.5)$ & \\
\hline 13-20.9 & $22,406(21.5)$ & $16,603(80.4)$ & 4039 (19.6) & & $554(2.7)$ & \\
\hline$\geq 21$ & $10,816(10.4)$ & 7697 (79.0) & $2048(21.0)$ & & $366(3.7)$ & \\
\hline $\begin{array}{l}\text { Charlson-Deyo } \\
\text { comorbidity score }\end{array}$ & & & & .001 & & $<.001$ \\
\hline 0 & $91,103(87.5)$ & $69,352(82.0)$ & $15,233(18.0)$ & & $2072(2.4)$ & \\
\hline 1 & $10,724(10.3)$ & $8039(80.4)$ & 1958 (19.6) & & $296(2.9)$ & \\
\hline$\geq 2$ & 2291 (2.2) & 1566 (76.2) & $490(23.8)$ & & $99(4.8)$ & \\
\hline Geography & & & & .323 & & .524 \\
\hline Rural & $2001(2.0)$ & 1515 (80.9) & $357(19.1)$ & & $46(2.4)$ & \\
\hline Urban & 15,179 (14.6) & $11,600(82.1)$ & 2533 (17.9) & & $343(2.4)$ & \\
\hline Metropolitan & 84,161 (80.8) & 63,376 (81.6) & $14,250(18.4)$ & & 2004 (2.6) & \\
\hline Ireatment facility & & & & $<.001$ & & $<.001$ \\
\hline Nonacademic & $51,511(49.5)$ & $39,691(84.7)$ & 7153 (15.3) & & $1014(2.2)$ & \\
\hline Academic & $42,434(40.8)$ & 30,827 (77.6) & 8907 (22.4) & & $1227(3.1)$ & \\
\hline
\end{tabular}

${ }^{1} 1414$ cases were missing for insurance status.

b10,173 cases were missing for academic/nonacademic facilities. 
$\mathrm{OR}=0.829)$. Compared with patients residing in zip codes where a high percentage of the population had high school degrees, areas with higher nongraduate rates had greater overall rates of MTD $(P<.001)$. Patients with more $\mathrm{CD}$ comorbidities also held an association with mMTD (CD1 with reference: CD0; $P=.011 ; \mathrm{OR}=1.080)(\mathrm{CD} 2$ with reference: $\mathrm{CD} 0 ; P<.001$; $\mathrm{OR}=1.364)$ and sMTD (CD2 with reference: CD0; $P<.001 ; \mathrm{OR}=1.877)$. Academic facilities had greater rates of mMTD (reference: nonacademic facilities; $P<.001 ; \mathrm{OR}=1.578)$ and $\mathrm{sMTD}(P<.001 ; \mathrm{OR}=1.366)$. In reference to head/neck primaries, primary sites on the trunk and extremities showed fewer mMTD (trunk: $P<.001, \mathrm{OR}=0.620$; extremities: $P<.001$, $\mathrm{OR}=0.641$ ) and sMTD (trunk: $P<.001, \mathrm{OR}=0.540$; extremities: $P<.001, \mathrm{OR}=0.632$ ). Compared with in situ disease, stage I melanomas were less likely to have treatment delay (mMTD: $P<.001, \mathrm{OR}=0.902$; sMTD: $P<.001, \mathrm{OR}=0.690$ ), whereas stages II (mMTD: $P<.001$, $\mathrm{OR}=1.130$ ), III (mMTD: $P<.001$, OR=1.196; sMTD: $P=.023, \mathrm{OR}=1.204$ ), and IV (mMTD: $P<.001, \mathrm{OR}=1.690$; sMTD: $P<.001, \mathrm{OR}=2.240$ ) were more highly associated with treatments delays.

\section{Comment}

The path to successful melanoma management involves 2 timeframes. One is time to diagnosis and the other is time to treatment. With $24.2 \%$ of patients receiving treatment later than 45 days after diagnosis, MTD is common and, according to our results, has increased on average from 2004 to 2015. This delay may be partially explained by a shortage of dermatologists, leading to longer wait times and follow-up. ${ }^{13,14}$ Melanoma treatment delay also varied based on insurance status. Unsurprisingly, those with private insurance showed the lowest rates of MTD. Those with no insurance, Medicare, or Medicaid/other government insurance likely faced greater socioeconomic barriers to health care, such as coverage issues. ${ }^{15}$ Transportation, low health literacy, and limited work schedule flexibility have been described as additional hurdles to health care that could contribute to this finding. ${ }^{16,17}$ Similarly, nonwhite patients, Hispanic patients, and those from zip codes with low high school graduation rates had more MTD. Although these findings may be explained by socioeconomic barriers and heightened distrust of the health care system, it also is important to consider physician accessibility. ${ }^{18,19}$

TABLE 4. Univariate $\chi 2$ Analysis of Tumor Characteristics and Melanoma Treatment Delay

\begin{tabular}{|c|c|c|c|c|c|c|}
\hline \multirow[b]{2}{*}{ Tumor characteristic } & \multicolumn{6}{|c|}{ Frequency, n (\%) } \\
\hline & $\begin{array}{l}\text { All cases } \\
(\mathrm{N}=104,118)\end{array}$ & $\begin{array}{l}\text { Delay of } \\
\text { treatment, } \leq 45 \mathrm{~d} \\
(\mathrm{n}=78,957)\end{array}$ & $\begin{array}{l}\text { Delay of } \\
\text { treatment, }>45 d \\
(n=17,681)\end{array}$ & $P$ value & $\begin{array}{l}\text { Delay of } \\
\text { treatment, }>90 d \\
(n=2467)\end{array}$ & $P$ value \\
\hline Primary site ${ }^{a}$ & & & & $<.001$ & & $<.001$ \\
\hline Head/neck & 33,195 (31.9) & $23,475(76.7)$ & 7133 (23.3) & & $1085(3.5)$ & \\
\hline Trunk & $27,024(26.0)$ & 21,231 (84.9) & 3768 (15.1) & & $434(1.7)$ & \\
\hline Extremities & $42,559(40.8)$ & 33,199 (83.8) & $6421(16.2)$ & & $860(2.2)$ & \\
\hline ge & & & & $<.001$ & & $<.001$ \\
\hline In situ & $26,819(25.8)$ & $19,972(80.6)$ & $4813(19.4)$ & & $700(2.8)$ & \\
\hline I & $43,139(41.4)$ & $34,266(84.1)$ & 6494 (15.9) & & $684(1.7)$ & \\
\hline ॥ & $13,883(13.3)$ & $10,362(79.5)$ & $2680(20.5)$ & & $414(3.2)$ & \\
\hline III & $9696(9.3)$ & $7190(78.7)$ & 1949 (21.3) & & 303 (3.3) & \\
\hline IV & $2703(2.6)$ & $1337(72.4)$ & $509(27.6)$ & & $140(6.5)$ & \\
\hline $\begin{array}{l}\text { uslow depth of } \\
\text { invasion, } \mathrm{mm}^{\mathrm{b}}\end{array}$ & & & & $<.001$ & & $<.001$ \\
\hline$<1$ & $43,551(41.8)$ & $34,279(84.0)$ & $6546(16.0)$ & & $783(1.9)$ & \\
\hline $1-1.9$ & $14,560(14.0)$ & $11,343(82.9)$ & $2342(17.1)$ & & $292(2.1)$ & \\
\hline $2-2.9$ & $6198(6.0)$ & $4684(80.7)$ & 1123 (19.3) & & $152(2.6)$ & \\
\hline $3-3.9$ & 3341 (3.2) & $2462(79.4)$ & 638 (20.6) & & $83(2.7)$ & \\
\hline $4-4.9$ & $2528(2.4)$ & $1828(77.5)$ & $530(22.5)$ & & 85 (3.6) & \\
\hline$\geq 5$ & $5372(5.2)$ & 3666 (73.8) & $1300(26.2)$ & & $232(4.6)$ & \\
\hline $\begin{array}{l}\text { Abbreviation: NOS, not o } \\
\text { a1340 coded as Skin, NC } \\
\text { b28,568 cases were miss }\end{array}$ & $\begin{array}{l}\text { vise specified. } \\
\text { Breslow data. }\end{array}$ & & & & & \\
\hline
\end{tabular}


Considering the 2011 Affordable Care Act along with the 2014 Medicaid expansion, our study holds implications on the impact of these legislations on melanoma treatment. Studies have supported expected rises in Medicaid coverage. ${ }^{20,21}$ The overall uninsured rate in the United States declined from $16 \%$ in 2010 to $9.1 \%$ in $2015 .{ }^{22}$ In our study, the uninsured population showed the highest average MTD rates, though those with Medicaid also had significant MTD. Another treacherous hurdle for patients is the coordination of care among dermatologists, oncologists, general surgeons, plastic surgeons, and Mohs surgeons as a multidisciplinary team. Lott et $\mathrm{al}^{6}$ found that patients who received both biopsy and excision from a dermatologist had the shortest treatment delays, whereas those who had a dermatologist biopsy the site and a different surgeon-including Mohs surgeons-excise it experienced significantly greater MTDs (probablility of MTD $>45$ days was 31\% [95\% CI, 24\%-37\%]. This discordant care and referrals could explain the surprising finding that treatment at an academic facility was independently associated with more MTD, possibly due to the care transitions and referrals that disproportionately affect academic centers and multidisciplinary teams, as mentioned above, regarding the transition of care to other physicians (eg, plastic surgeon). A total of $70.1 \%$ of our cases treated at academic facilities reported a prior diagnosis at another facility. These results should not dissuade the pursuit of multidisciplinary treatment teams but should raise caution to untimely referrals.

Age, sex, and race were all associated with more MTD. Patients older than 50 years likely face more complex decisions regarding treatment burden, quality of life, and functional outcomes of more aggressive treatments. High rates of surgical refusal for a number of malignancies have been documented in the elderly population, ${ }^{23-25}$ which is of particular concern for the high surgery burden of head and neck melanomas, ${ }^{26}$ as further supported by the findings of more MTD for head and neck primaries. As with elderly patients, patients with higher comorbidity scores and more advanced tumors face similar family-patient care discussions to guide treatment. Additionally, women were more likely to experience MTD, which may be connected to a greater concern for cosmesis ${ }^{27}$ and necessitate more complex management options, such as Mohs micrographic surgery (a procedure that has gained some support for melanoma excision with the help of immunostaining). ${ }^{28}$

There are several limitations to this study. Accurate data rely on precise record keeping, reporting, and coding by the contributing institutions. The NCDB case diagnosis is derived from data entry without a centralized review process by experienced dermatopathologists. We could not assess the effects of tumor diameter, as these data were inadequately recorded within the dataset. The NCDB also does not provide details on specific immunotherapy or chemotherapy agents. The NCDB also is a facilitybased data source, potentially biasing the melanoma data toward thicker advanced tumors more readily managed at such institutions. Lastly, it is impossible to distinguish between patient-related (ie, difficult decision-making) and health care-related (ie, health care accessibility) delays. Nonetheless, we maintain that minimizing MTD is important for survival outcomes and for limiting the progression of melanomas, regardless of the underlying rationale. We believe that our study expands on conclusions previously limited to a Medicare population.

\section{Conclusion}

According to the NCDB, mean MTD has increased significantly from 2004 to 2015. Our results suggest that MTD is relatively common in the United States, thereby increasing the risk for metastases. Higher MTD rates are independently associated with being older than 50 years, female, nonwhite, not privately insured, Hispanic, and treated at an academic facility; having a positive comorbidity history and stage II to IV tumors; and residing in a zip code with a low high school graduation rate. Stage I tumors, primaries not located on the head or neck, and residing in a zip code with a higher median income are associated with lower MTD rates. Policymakers, patients, and dermatologists should better recognize these risk factors to facilitate patient guidance and health equity.

\section{REFERENCES}

1. Huff LS, Chang CA, Thomas JF, et al. Defining an acceptable period of time from melanoma biopsy to excision. Dermatol Reports. 2012;4:E2.

2. Matthews NH, Li WQ, Qureshi AA, et al. Epidemiology of Melanoma. Cutaneous Melanoma: Etiology and Therapy. Codon Publications; 2017.

3. Siegel RL, Miller KD, Jemal A. Cancer statistics, 2017. CA Cancer J Clin. 2017;67:7-30.

4. Nelson BR, Hamlet KR, Gillard M, et al. Sebaceous carcinoma. J Am Acad Dermatol. 1995;33:1-15.

5. Fan Q, Cohen S, John B, et al. Melanoma in situ treated with topical imiquimod for management of persistently positive margins: a review of treatment methods. Ochsner J. 2015;15:443-447.

6. Lott JP, Narayan D, Soulos PR, et al. Delay of surgery for melanoma among Medicare beneficiaries. JAMA Dermatol. 2015;151:731-741.

7. Renzi C, Mastroeni S, Mannooranparampil TJ, et al. Delay in diagnosis and treatment of squamous cell carcinoma of the skin. Acta Derm Venereol. 2010;90:595-601.

8. Winchester DP, Stewart AK, Phillips JL, et al. The National Cancer Database: past, present, and future. Ann Surg Oncol. 2010;17:4-7.

9. Raval MV, Bilimoria KY, Stewart AK, et al. Using the NCDB for cancer care improvement: an introduction to available quality assessment tools. J Surg Oncol. 2009;99:488-490.

10. Turkeltaub AE, Pezzi TA, Pezzi CM, et al. Characteristics, treatment, and survival of invasive malignant melanoma (MM) in giant pigmented nevi (GPN) in adults: 976 cases from the National Cancer Data Base (NCDB). J Am Acad Dermatol. 2016;74:1128-1134.

11. Boffa DJ, Rosen JE, Mallin K, et al. Using the National Cancer Database for outcomes research: a review. JAMA Oncol. 2017;3:1722-1728.

12. Riker AI, Glass F, Perez I, et al. Cutaneous melanoma: methods of biopsy and definitive surgical excision. Dermatol Ther. 2005;18:387-393.

13. Kimball AB, Resneck JS Jr. The US dermatology workforce: a specialty remains in shortage. J Am Acad Dermatol. 2008;59:741-745.

14. Glazer AM, Farberg AS, Winkelmann RR, et al. Analysis of trends in geographic distribution and density of US dermatologists. JAMA Dermatol. 2017;153:322-325. 
15. Okoro CA, Zhao G, Dhingra SS, et al. Peer reviewed: lack of health insurance among adults aged 18 to 64 years: findings from the 2013 Behavioral Risk Factor Surveillance System. Prev Chronic Dis. 2015;12:E231.

16. Syed ST, Gerber BS, Sharp LK. Traveling towards disease: transportation barriers to health care access. J Community Health. 2013;38:976-993.

17. Valerio M, Cabana MD, White DF, et al. Understanding of asthma management: Medicaid parents' perspectives. Chest. 2006;129:594-601.

18. Kaplan CP, Nápoles A, Davis S, et al. Latinos and cancer information: perspectives of patients, health professionals and telephone cancer information specialists. J Health Dispar Res Pract. 2016;9:154-167.

19. Armstrong K, Ravenell KL, McMurphy S, et al. Racial/ethnic differences in physician distrust in the United States. Am J Public Health. 2007; 97:1283-1289.

20. Moss HA, Havrilesky LJ, Chino J. Insurance coverage among women diagnosed with a gynecologic malignancy before and after implementation of the Affordable Care Act. Gynecol Oncol. 2017;146:457-464.

21. Moss HA, Havrilesky LJ, Zafar SY, et al. Trends in insurance status among patients diagnosed with cancer before and after implementation of the Affordable Care Act. J Oncol Pract. 2018;14:E92-E102.
22. Obama B. United States health care reform: progress to date and next steps. JAMA. 2016;316:525-532.

23. Crippen MM, Brady JS, Mozeika AM, et al. Impact of body mass index on operative outcomes in head and neck free flap surgery. Otolaryngol Head Neck Surg. 2018;159:817-823.

24. Verkooijen HM, Fioretta GM, Rapiti E, et al. Patients' refusal of surgery strongly impairs breast cancer survival. Ann Surg. 2005;242:276-280.

25. Wang J, Wang FW. Refusal of cancer-directed surgery strongly impairs survival of patients with localized hepatocellular carcinoma. Int J Surg Oncol. 2010;2010:381795.

26. Zito PM, Scharf R. Cancer, melanoma, head and neck. StatPearls. StatPearls Publishing; 2018.

27. Al-Dujaili Z, Henry M, Dorizas A, et al. Skin cancer concerns particular to women. Int J Womens Dermatol. 2017;3:S49-S51.

28. Etzkorn JR, Jew OS, Shin TM, et al. Mohs micrographic surgery with melanoma antigen recognized by $\mathrm{T}$ cells 1 (MART-1) immunostaining for atypical intraepidermal melanocytic proliferation. J Am Acad Dermatol. 2018;79:1109-1116.e1 